\title{
ASSESSMENT OF MAGNETIC FIELD ASYMMETRIES IN ELMO BUMPY SQUARE"
}

\author{
T. UCKAN and N. A. UCKAN \\ Oak Ridge National Laboratory \\ Oak Ridge, Tenuessee 37831
}

$4 \times \equiv 2000$

(615) $574-1354$

\begin{abstract}
There exist two separate and independent magnetic field asymmetries in the ELMO Bumpy Square (EBS). One is associated with the small perturbations in the magnetic field, known as the field errurs, caused by coil misalignments during installation, imperfection in coil winding, etc. The second source of asymmetry is the magnetic field ripple in the nigh-field toroidal solenoids (ccrners) produced by the finiteness of the number of coils. In general, these two sources of asymmetry introduce enhanced transport losses (in addition to other effects) to the system, alt ugh they affect different classes of particles. Toroidally pass - 6 (circulating) particles $\left(v_{1} / v-1\right)$ are influenced by the field errors, whereas trapped particles $\left(v_{1} / v \sim 0\right)$ in the corners are influenced by the field ripple. In this paper we discuss these two effects separately and calculate the allowable magnitudes of the field error and field ripple in EBS, both for an experimentaj-size device and for a reactor.
\end{abstract}

\section{INTRODUCTION}

The ELMO Bumpy Square (EBS) geometry consists of linear segments of simple mirrors that are linked by sections of high-field coroidal solenoids (corners) as shown in Fig. 1. In this configuration the tomidal effects are localized in the corners. 1.2

An ideai EBS is a closed field line configuration with zern rotational transform and no toroidal current, characteristies of an ideal ELMO Bumpy Torus (EBT). This current-free equilibrium configuration is very sensitive to the system magnetic field asymmetries (field errors). If large enough, these field errors $(\delta B / B)$ can cause field lines to spiral out of the confinement volume (enbanced losses), thereby degrading confinement. 3.4 In practice, the field errors can arise from many different sources: coil mikalignment, imperfect coil winding, the presence of nearby magnetic materials, etc. In this paper we discuss only the first error source and

\footnotetext{
"Research spoesored by the Office of Fusion Enery, U.S. Depertment of Eneray, under Contract No. DE-ACO5-84OR21400 with Martin Marietta Energy Systema, Inc.
}

ORNL-DWG 84-2753A FED

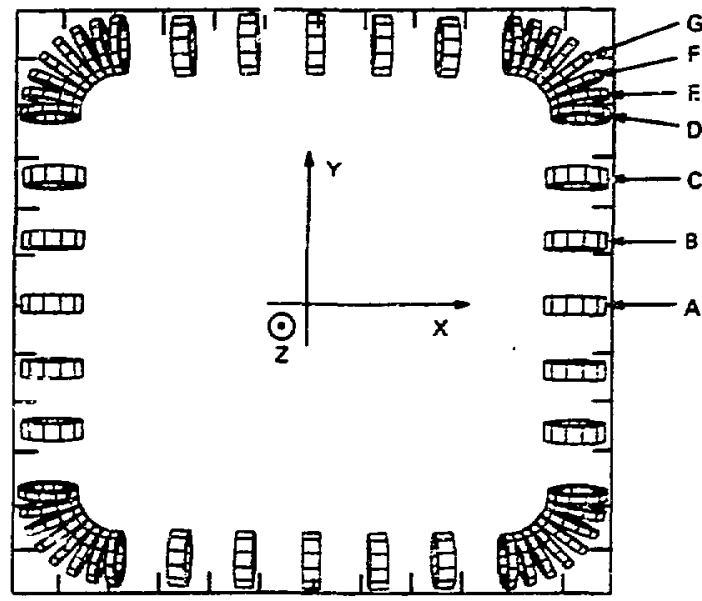

Fiz. 1. Geometry of an EBS configuration indicating the coil arrangements (top view). Coordinate system used is shown ( $X$ and $Y$ axes are indicated; $Z$ axis points out of the viewing area). There are four symmetry planes and eight field periods. One field period is represento by coils A through $\mathbf{G}$.

estimate the maximum tolerable field error $(\delta B / B)$ and, in tum, the corresponding constraint imposed on the alignment of the coils.

Numerically calculated transport coefficients indicate that the neoclassical confinement tisae should be a factor of 5 to 15 larger [depending on the global mirror ratio $M_{G}=$ $B_{\text {octer }} / B_{\text {sids }}$ (midplanc)] ior the EBS than for an EBT of comparable size. In order for neoclassical losses to be dominant, ripple-induced loses from the toroidal solenoind sections shou'd be smaller than the neoclessical loses from the straight sections. The ripple is produced by the finitcaess of 
the number of coils in the corners. The presence of ripple will introduce additional particle trapping and, if large enough, may be the primary factor determining the transport of particles and energy in the region of low collision frequencies. ${ }^{3}$ In the phenomenon known as ripple trapping, the particles become trapped is the freld minimum between coils (between coils which produce the toroidal solenoid field in the corners) that are localized in the toroidal direction and experience a unidirectional toroidal drift thet leads to the ripple diffusion. A similar phenomenon occuss in other toroidal configurations (e.g., tokamaks). In this paper we calculate the enhanced transport coefficients aseociated with the ripple-induced drifts and compare them with the EBT neoclassical diffusion coefficients to determine the allowable range for the magnitude of the field ripple.

In our examples, for an experimental-size device, we consider an EBS configuration (Fig. 1) whose sides are constructed from EBT-Scale (EBT-S) mirror coils (with five mirror coils per side, such as $A, B$, and $C$ ) and whose corners are $90^{\circ}$ sections of a toroidal solenoid in which the field is produced by (1) four EBT-S mirror coils or (2) eight half-size EBT-S coils (such as D, E, F, and G) per corner.

\section{FTELD ERROR CALCULATIONS}

As pointed out, the field errors can arise from many different sources: coil misalignment, imperfect winding effeets in a coil, magnetic fields from busworl and leads, and field perturbatiots due to the presence of magnetic materials. Although all these sources can cause significant error fields, we will discuss only the first error source in this paper.

The presence of field errors introduces an additional vertical drift of order $v_{f}(\delta B / B)$ to the system. Mirror-trapped particles $\left(v_{1} / v-0\right)$ are not significantly affected (to lowest order) by field errors because their motion is restricted to a single sector. ${ }^{3-4}$ The drift orbits of toroidally passing particles $\left(v_{1} / v-1\right)$, on the other hand, are modified by this additional drift, winich, if it is large enough, causes them to walk out of the onsfinement system. We define a critical error field $(\delta B / B)_{\text {crit }}$ such that if $(\delta B / B)>(\delta B / B)_{\text {erit }}$ the drift surfaces of toroidally passing particles become open. From the requirement of hoving closed drift surfaces, ${ }^{3,4}$ it is necessary to have $(\delta B / B)<(\delta B / B)_{\text {crit }} \sim p_{e} /\left\langle R_{c}\right\rangle$, where. $p_{e}$ is the electron Larmor radius and $\left\langle\boldsymbol{R}_{c}\right\rangle$ is the average magnetic radius of curvature. For an experimental-size device (a device similar in size to EBT-S), the required $(\delta B / B)_{\text {erit }}$ few $\times 10^{-4}$, and for a reactor-like device $(\delta B / B)_{\text {erit }} \leqslant$ $10^{-5}$. We note that the field errors will be more critical in regions of large magnetic field and large radii of curvature $\left[(\delta B / B)-p / R_{c}-\left(B R_{c}\right)^{-1}\right]$, that is, near the coil throat and especialiy at the magnetic axis. "Thus, we will evaluate the amount of $\delta \vec{R}$ that a field line centered at the magnetic axis (starting in the coil throat) misses connectiog with itself when followed once around the torus. For perfect coils installed with no misalignment, this number should be zero.

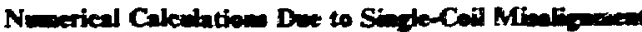

Shown in Fig. 1 is the geometry of an FBS configuration indicating the coil arrangements and condinate systen used. An EBS configuration has four symmetry planes and eigbt field periods. Specific coils involved in the calculations within one field period are deagonted $A$ thmoge $O$ in Fig. 1. Figure 2 sbows magnetic field lines in the equatorial plane ( $X-Y$ plane) with specific dimensions. The mirror sector length (or the coil spacing) in the sides is $L_{N 1}=40 \mathrm{~cm}$, and the major radius of the corner sections is $R_{\text {conar }}=$ $44 \mathrm{~cm}$, with the axis of each corner section dispiaced radially outward by ( $\Delta$ shift) $=2.5 \mathrm{~cm}$ irom the axis of the sides. This displacement and the length of the transition sector $\left(L_{T f}\right)$ are adjusted so that the rings in the transition sector (the sector connecting the simple mirror field to a $1 / R$ varying toroidal field) form on the same flux lines as in the axisymmetric sectors. Depending on the ratio of the carrents in the corner coils to the currents in the straight section, the transition sector length is typically larger $(-2$ to $4 \mathrm{~cm})$ than the length of the mirror sectors. For $I_{\text {corner }} / I_{\text {side }}=1.2-1.4$, $L_{T_{\mathrm{r}}}=42-44 \mathrm{~cm}$. In our example, we bave $I_{\text {consen }} / I_{\text {ide }}=1.4$ and $L_{\mathrm{Tt}}=42 \mathrm{~cm}$.

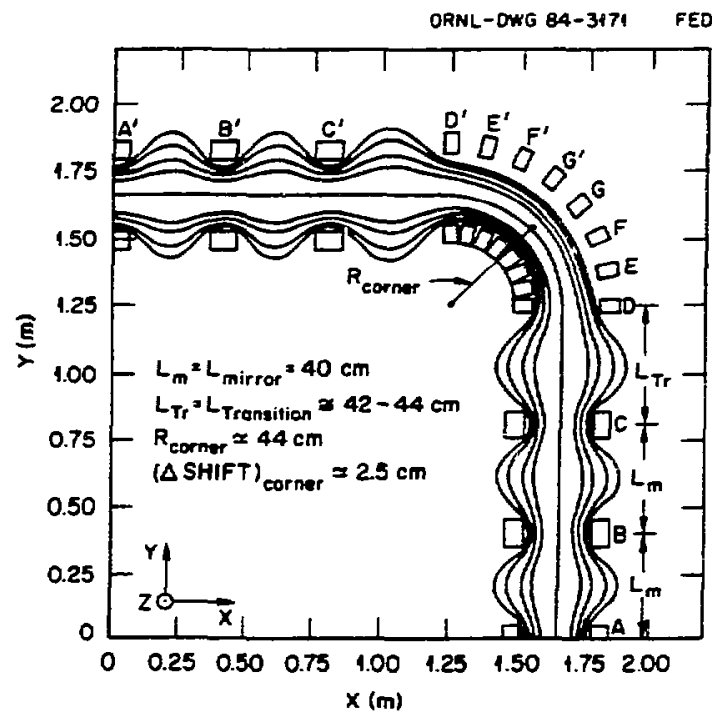

Fig. 2. Magnetic field lines in the equatorial plane ( $X-Y$ plane) of an EBS are showr! for one quadrant. A through $G$ coils and their mirror images $G$ through $A^{\prime}$ are shown along with specific dimensions used in the calculations. Here $L_{m}=$ mirror sector length, $L_{T_{r}}=$ transition sector length, $k_{\text {oornet }}=$ radius of the corner, and ( $\Delta$ shift) lomer $=$ (center of coil D - center of coil A) $=X_{D 0}-X_{A 0}$. 
The field line geometry shown in Fig. 2 is calculated with finite coil dimensions from EFFI, which do not differ from those of circular filament approximation. ${ }^{2}$ The code evaluates $\delta \tilde{R}(\delta X, \delta Y, \delta Z)$ in a coil plane by integrating the field line equations through one full period around the machine $\left(360^{\circ}\right.$ in $\left.\phi\right)$. Theoretically, $\delta \vec{R}$ vanishes for the ideal positions and orientations of the coils. Actual numerical results obtained from the code usually range from $10^{-9}$ to $10^{-10} \mathrm{~cm}$ for $|\delta \vec{R}|$. Therefore, the accuracy of the cote seems sufficient for the purpose of the present study.

There are five degrees of freedom associated with each coil: three for the position of the coil center and two for the angles of the coil plane. The five corresponding coil errors are renoted by $\Delta X, \Delta Y, \Delta Z, \Delta \theta$, and $1 \phi$, where $\theta$ is the angle between the $Z$ axis and the projection of the coil nor$\mathrm{mal}$ to the $X-Y$ plane and $\phi$ is the angle between the coil plane (which is coplanar with the $Z$ axis) and the $X$ axis in the $X-Y$ plane (Fig. 3 ).

Table I lists the field line displasemen: $\delta \hat{R}^{\text {v }}$ obtained with only one coil perturbod and with only the error involved in coil position or orientation. The freld line displacement is measured in the center of the coil plane of the $A$ coil $(X-Z$ plane with coil center at $X-X_{\text {An }} Y=0, Z=Z_{10}=0$ ). Figures 4 and 5 show the direction and relative magaitude of field line displacement in a cirnle with a radius of $11 \mathrm{~cm}$ (the radius of the clear bore under the coil) in the coil plane for $\Delta \theta=1^{\circ}$ and $\Delta \phi=1^{\circ}$, respectively.

The results of the calculations indicate that the field line closure is most sensitive to anguiar misalignment of the coils ( $\Delta \theta$ and $\Delta \phi$ ). In these cases, an assumed angular error of $1^{\circ}$ causes $\delta B / B=2.22 \times 10^{-4}$. We note that in EBT-S a similar angular misaligament of $1^{\circ}(\Delta \theta$ or $\Delta \phi$ ) causes similar error fields $\left(\delta B / B=2.22 \times 10^{-4}\right)$. An impotant observation is that the effects of errors in $\theta$ and $\phi$ are escentially orthogonal: $\Delta \theta$ causes a vertical displacement of the field
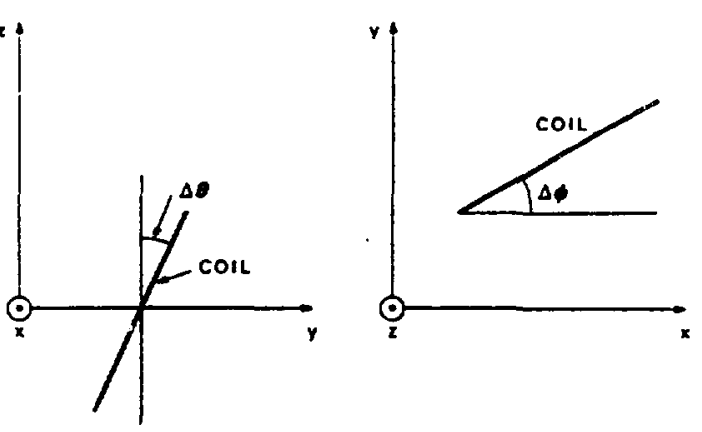

Fig. 3. Two angies of the coil plane. Orientations of $\mathbf{X}, \mathbf{Y}$, and $Z$ coordinates are shown, with the same orientation as in Figs. $I$ and 2. lines (iig. 4) and $\Delta \phi$ causes a horizontal displacement of the field lines (Fig. 5). Errors in absolute positions are much less sensitive; a misalignment of $1 \mathrm{~cm}$ in one coil location yields an error $\delta B / B-10^{-6}$ to $10^{-7}$ (Table I).

\section{Statistical handysio of Errors}

In the previous section, we determined that the dominant misalignment contributions to field erms were in the two angles $\theta$ and $\phi$. Since the proper determination of angular

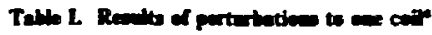

\begin{tabular}{|c|c|c|c|c|}
\hline \multirow[b]{2}{*}{ Coil } & \multirow[b]{2}{*}{ Perturbetioo } & \multicolumn{2}{|c|}{$\begin{array}{l}\text { Field line displecemeat } \\
(d R)\end{array}$} & \multirow[b]{2}{*}{$18 / 8$} \\
\hline & & $\begin{array}{c}\delta X \\
(\min )\end{array}$ & $\begin{array}{c}\delta Z \\
(\mathrm{~mm})\end{array}$ & \\
\hline $\mathbf{A}$ & $\Delta-1^{\circ}$ & $\Rightarrow 0$ & $-2 B 2$ & $222 \times 10^{-4}$ \\
\hline $\mathbf{A}$ & $\Delta \phi=1^{\circ}$ & -281 & $\Rightarrow 0$ & $2.22 \times 10^{-6}$ \\
\hline $\mathbf{G}$ & $\Delta-1^{\circ}$ & $\Rightarrow 0$ & $-i .1 y$ & $9.22 \times 10^{-5}$ \\
\hline $\mathbf{G}$ & $\Delta \phi=1^{\circ}$ & -1.21 & 0 & $9.54 \times 10^{-5}$ \\
\hline $\mathbf{B}$ & $\Delta x=1 \mathrm{~cm}$ & $1.1 \times 10^{-2}$ & 0 & $8.67 \times 10^{-7}$ \\
\hline $\mathbf{B}$ & $\Delta Y=1 \mathrm{~cm}$ & $6.2 \times 10^{-3}$ & 0 & $4.89 \times 10^{-1}$ \\
\hline $\mathbf{B}$ & $\Delta Z=1 \mathrm{~cm}$ & $\mathbf{0}$ & $1.7 \times 10^{-2}$ & $1.34 \times 10^{-6}$ \\
\hline $\mathbf{B}$ & $\Delta / / I=10^{-3}$ & $3.4 \times 10^{-4}$ & 0 & $268 \times 10^{-1}$ \\
\hline
\end{tabular}

Fiekd line displacement is measured in the center of the $A$ coil plane $(X-Z$ plane). Calculated lact of field line clowre $\delta f(b X, \delta Z)$ and exrespoadine field erroe $8 B / B=\mid S R / C$. where $C$ is the circumference (field line length).

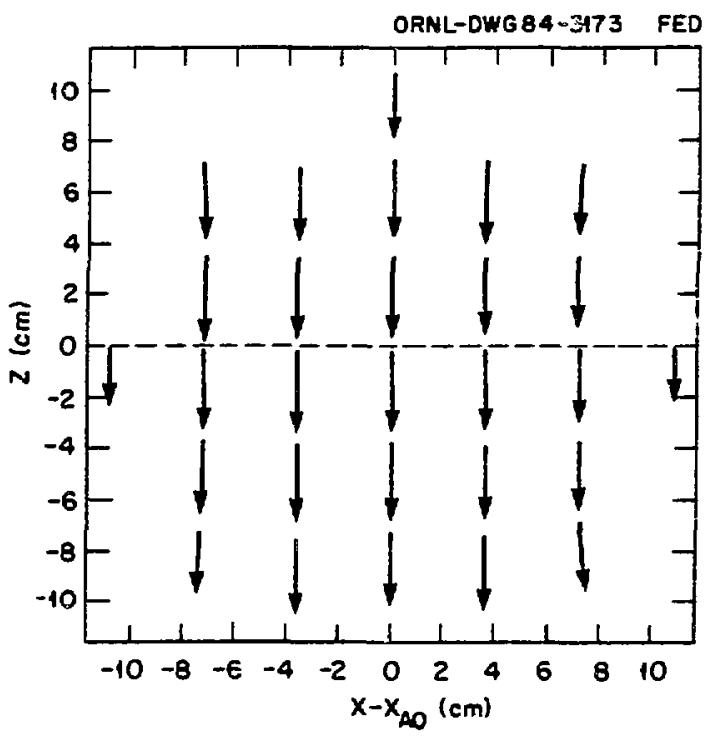

Fig. 4. Arrow plot showing the direction and relative magnitude of field line displacements in the plane of $A$ coil for $\Delta \theta=1^{\circ}$. The largest magnitude is $2.82 \mathrm{~mm}$ at the coil center $(\mathbf{0 , 0})$. 


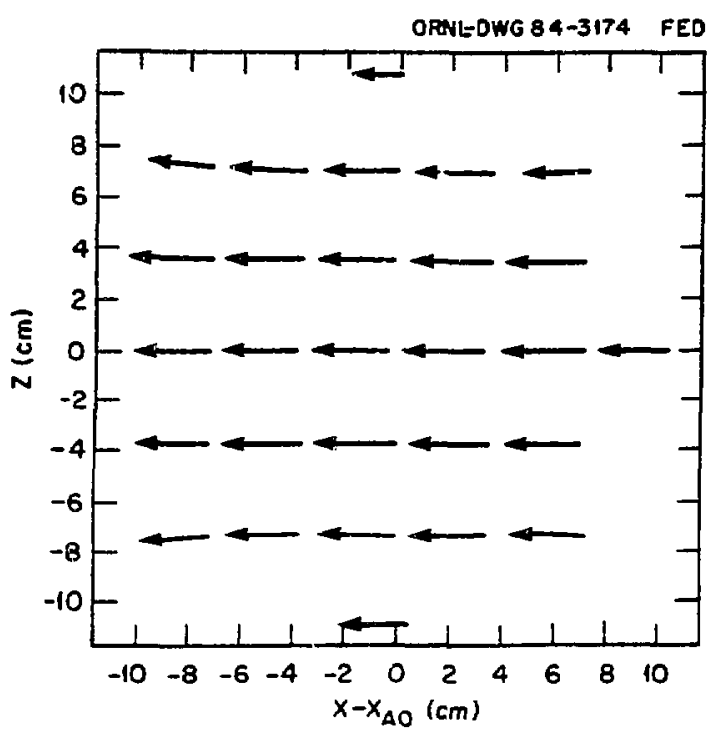

Fig. 5. Arrow plot showing the direction and relative magnitude of field line tisplacements in the plane of $A$ coil for $\Delta \phi=1^{\circ}$. The largest magnitude is $2.81 \mathrm{~mm}$ at the coil center $(0,0)$.

aligament will require fairly stringent positional alignment also, we discuss only the angular errors and assume that nonclosure of field lines arising from positioning errors is small relative to the angular ones.

First, we consider only the variations in $\theta$. The total number of coils in the sides is $N_{s}=20$ (five coils per side) and in corners $N_{c}=32$ (eight coils per corner). For one coil (side or corner), we have the expression $\delta B / B=C_{0} \Delta \theta$. If we assume that the errors in all $\mathbf{2 0}$ straight-side coils are distributed normally with one Gaussian distribution and that the errors in all 32 corner coils are distributed normally with anuther Gasssian distribution (both of which have a mean error of zero and an rms error of $\theta_{\text {rms }}$ ), then the problem is exactly equivalent to the one-dimensional random walk problem. Each magnet arror $\Delta \theta$ contributes a step-size $(\delta Z)$, to a field line, where $j=s(s i d e)$, c(corner). For small individual displacements, the problem can be treated as a linear superposition of steps of varying size and direction with an rms step-size determined by $\theta_{\text {tmas }}$. For $N$ steps, the rms displacement $\delta Z_{N}=N^{1 / 2} \delta Z_{1}$; then,

$$
\begin{aligned}
& (\delta B / B)_{0}=(\delta B / B)_{\text {coner }}+(\delta B / B)_{\text {side }} \text {, }
\end{aligned}
$$

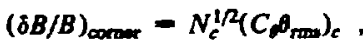

$$
\begin{aligned}
& (\delta B / B)_{\text {side }}=N_{s}^{1 / 2}\left(C_{\theta} \theta_{\mathrm{rm}}\right)_{s} .
\end{aligned}
$$

Thus,

$$
(\delta B / B)_{\ell}=\left[(32)^{1 / 2} C_{k}+(20)^{1 / 2} C_{b s}\right]^{B} \mathrm{rm} .
$$

where $\left(\theta_{\mathrm{rma}}\right)_{c}=\left(\theta_{\mathrm{rm}}\right)_{z}$ is taken.

The same arguments apply for errors in the $\phi$ direction. Since the errors in $\phi$ and $\theta$ cause the same magnitude change in $\delta B / B$ (i.e., $C_{1}-C_{\text {b }}$ from Table $I$ ) bul are nerpendicular in direction, the expression for the tosal field error is simply

$$
(\delta B / B)=\left[(\delta B / B)^{2}+(\delta B / B)_{\phi}^{2}\right]^{1 / 2},
$$

which yields

$$
\delta B / B=\left[(32)^{1 / 2} C_{k}+(20)^{1 / 2} C_{\theta s}\right]\left(\theta_{n \pi}^{2}+\phi_{n \pi}^{2}\right)^{1 / 2} .
$$

where $\phi_{\mathrm{rms}}$ is the rms error in $\phi$. The previous expression is valid if the errors in $\phi$ and $\theta$ are uncorrelated, which sems to be the most reasonable assumption to make. Finally, if we assume the rms errors in the $\phi$ and $\theta$ directions to be equal, then

$$
\delta B / B=\left[8 C_{\theta c}+(40)^{1 / 2} C_{\theta s}\right] \theta_{\mathrm{mms}} .
$$

From Table I we have $C_{\theta c}=9.54 \times 10^{-5}$ and $C_{t s}=$ $2.22 \times 10^{-4}$. Thus, $\delta B / B=2.17 \times 10^{-3} \theta_{\mathrm{rms}}$, where $\theta_{\mathrm{rm}}$ is expressed in degrees.

The question remains, "What is the iaherent $\delta B / B$ that can be tolerated?" (That is, what is the field error inherent to the device construction without global correction?) In the EBT-S device the inherent field error was $\delta B / B=5 \times$ $10^{-4}$, which was corrected to a level of $\delta B / B=10^{-4}$ with the global field error correction coils. ${ }^{3,4}$ That is, the global field correction coils correct $80 \%$ of the inherent device field error in EBT.S. Based on the EBT-S experience ${ }^{4}$ and the fact that coil alignment errors produce fairly uniform field errors across the plasma (Figs. 4 and 5), we assume that similar levels of field errors $\left(\delta B / B=10^{-4}\right)$ will be required in ERS and that ihe global field error correction coils can correct $80 \%$ of the inherent device field error. Thus, the inherent field error criterion (that is, five times that for the net field error $\delta B / B=5 \times 10^{-4}$ ) will yield the required values for $\theta_{\mathrm{mm}}$. Solving

$$
\delta B / B=2.17 \times 10^{-3} \theta_{\mathrm{rmat}}=5 \times 10^{-4},
$$

we have $\theta_{\mathrm{rm}}=0.23^{\circ}$.

Since the treatment here is statistical in nature (as is the alignment of the coils), the actual $\delta B / B$ is not specified exactly by a specification of $\theta_{\text {rm. }}$. Rather, it has a Gaussian distribution; when corrected with global correction coils, the probability that $\delta B / B$ will be less than $10^{-4}$ is about $68 \%$ and the probability that it will be las than $2 \times 10^{-4}$ is more than 95\%. 
Because drift orbits in EBS are much better centered than those in EBT-S. ${ }^{1.2}$ the effect of field errors on particle orbit displacement might be expected to be more pronounced is EBS than in EBT.S. In this regard, if we choose to be more pessimistic in the field ertor criterion in EBS than in EBT.S, we can assume that the inherent field errot should not exceed $\delta B / B=2 \times 10^{-4}$ (instead of $\left.\delta B / B=5 \times 10^{-*}\right)$. Under this pessimistic assumption $\theta_{\text {mor }}$ (pessimistic) $=0.1^{\circ}$. With this value of $\theta_{\text {mos }}$ the probability that inherent $\delta B / B$ will be less than $4 \times 10^{-4}$ is greater than $95 \%$, and, when it is corrected, $\delta B / B$ is less than $8 \times 10^{-3}$

Repeating similar calculations for a typical EBS reactor, ${ }^{2}$ we find $\delta E / B=1.7 \times 10^{-3} \theta_{\mathrm{mr}}$. As noted earlisr, $(\delta B / B)_{\text {crit }} \leq 10^{-5}$. Again, assuming that the intrinsic error criterion is five times that for the net error field, $\delta B / B \leq$ $4 \times 10^{-5}$. Thus, for a reactor, the $\theta_{\text {ros }}$ required to obtain this value of $\delta B / B$ is $\theta_{\mathrm{rms}}=0.024^{\circ}$.

\section{RIPPLE EFFECTS}

In this section we consider the effect of toroidal field ripple (due to the discrete coils in the high-field corners) on transport In the presence of ripple, there is a new class of so-called locally trapped particles $\left(v_{t} / v-0\right)$ in the corners. These particles oscillate (trapped) in the field minimum (local magnetic well) between coils (those which produce the toroidal solenoid field in the corners) that are localized in the toroidal direction and experience a unidirectional toroidal drift that leads to the ripple-enhanced diffusion. Especially in the low-collisionality $(v / \Omega)$ regime, rippleinduced losses could lead to significant increases in diffusion and thermal conductivity and may also be the main factor determining the transport of particles and energy. We note that the ripple trapping affects only a relatively small group of particles, that is, those with $v_{y}$ (parallel velocity) so small that they can be trapped in the ripple.

In general, the ripple modulation $\delta$ (magnitude of field rippie) varies both radially and poloidally. The poloidal variation has the effect of reducing or completely eliminating the ripple well depth on the inside of the toroidal sections, with the precise degree of reduction depending on the shape of the coils and the position of the plasma within the coils. For simplicity, we will neglect the poloidal variations, with the result of overestimating the ripple effects.

\section{Magretic Field Molled}

Figures 6 and 7 show the magnetic field strength as a function of arc length along the magnetic axis of a four- and eight-coil per corner EBS, respectively. The on-axis mirror ratio in the sides is seen to be 1.9 , and the global mirror ratios [ $B_{\text {corver }} / B_{\text {tide }}$ (midplate)] for these particular cases are 3.4 and 3.85 , respectively. In buth cases the corner coils have the same total number of ampere-turns. Althcugh the field ripple is apparent for a four-coil per corner case (Fig. 6), the ripple is practically zeto for most of the plasma

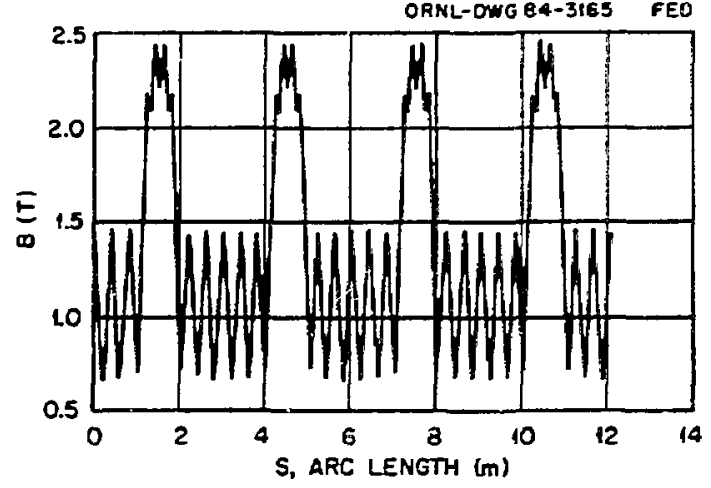

Fig. 6. Magnetic field strength as a function of are length along the magnetic axis for a four-coil per corner EBS.

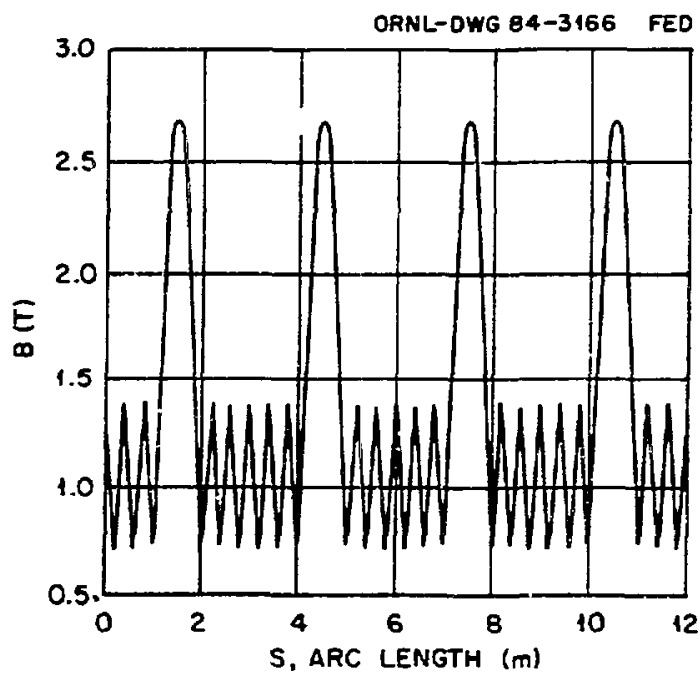

Fig. 7. Magnetic field strength as a function of are length along the magnetic axis for an eight-coil per comer EBS (Fig. 2).

cross section for an eight-coil per comer case (Figs, 2 and 7). A variation of field ripple as a function of normalized radius is shown in Fig. 8 for both cases and is summarized in Table II.

For high-field toroidal corners, a simple model for the magnetic field is

$$
\vec{B}=\frac{B_{o}}{1+\cos \theta}\left[\frac{B_{r}}{B_{\diamond}}, 0,1-\delta(r, \theta) \cos N \phi\right],
$$


OANL-DWG A4C.3167 FED

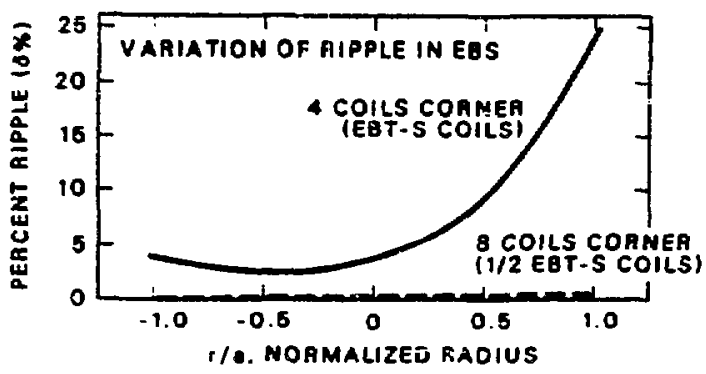

Fig. 8. Variation of ripple amplitude $\delta$ as a function of radius for (a) four coils per corner (solid tine) and (b) eight coils per corner (dashed line).

Table II. Ripple rariation in the corners of EBS

\begin{tabular}{ccc}
\hline & \multicolumn{2}{c}{ Ripple amplitudes (\%) } \\
\cline { 2 - 3 } $\begin{array}{c}\text { Normalized } \\
\text { radius } \\
r / a\end{array}$ & $\begin{array}{c}\text { Four-coil/ } \\
\text { corner }\end{array}$ & $\begin{array}{c}\text { Eight-coil/ } \\
\text { corner }\end{array}$ \\
\hline-1.0 & 3.5 & 0 \\
-0.5 & 2.5 & 0 \\
0.0 & 4.0 & 0 \\
+0.5 & 8.5 & -0 \\
+1.0 & -25.0 & $\sim 0.5$
\end{tabular}

"Here $a$ is the last field line that just grazes the coil throat in the straight sections ( $a \Rightarrow$ $11 \mathrm{~cm})$.

where $r$ and $\theta$ an polar coordinates in the minor cross section of the corners, $\phi$ is the angular coordinate (toroidal angle) along the magnetic axis of the corners, $\epsilon=r / R_{0}$ is the inverse aspect ratio of the toroidal corner (with $R_{0}$ the major radius of the corner), $N$ is the number of coils in the corners (a four-coil per corner case corresponds to an $N=$ 12 coil torus and an sight-coil per corner case corresponds to an $N=28$ coil torus), and $\delta(r, \theta)$ is the ripple well depth (modulation), defined as

$$
\delta(r, \theta)=\left|\frac{B_{\max }-B_{\text {tin }}}{B_{\max }+B_{\min }}\right\rangle_{\text {corror }}=\left|\frac{\Delta B}{B_{0}}\right\rangle_{\text {comper }} .
$$

The radial component of $\vec{B}$, necessary to satisfy $\vec{\nabla} \cdot \vec{B}=0$, is smell (on the order of $B_{r} \sim \delta B_{0}$ sin $N \phi$ ). Thus, the field strength in the corner is approximately

$$
B=B_{\phi}=B_{o}[1-\epsilon \cos \theta-\delta(r, \theta) \cos N \phi] .
$$

\section{Trealud and Critical Energies (Ref. 5)}

The threshold energy is defined as the energy above which particles execute more than one bounce motion in a ripple before beins scattered out of the loss region $\left(v_{1}<\delta^{1 / z_{1}}\right.$ ) associated with ripples (Fig. 9). This enercy can be obtained

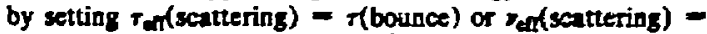
(bounce), where $\nu_{e t}=y_{\varphi_{0}} /(\Delta \theta)^{2}=\nu_{x_{0}} / \delta$ and $\alpha($ bounce $)=$ $\delta^{1 / 2} v / L=\delta^{1 / 2} v N /\left(2 \pi R_{0}\right)$. Here ${ }^{2}, 0$ is the $90^{\circ}$ colliaion fre quency, $v$ is the particle speed, $L$ is the length of the ripple well $\left(L-2 \pi R_{0} / N\right)$, and the fraction of particles that are ripple trapped is equal to $(\delta)^{1 / 2}$. Combining all gives the threshold energy (temperature) as (in mks units with temperature in electron-volts)

$$
T_{\mathrm{tb}}=90 \delta^{-3 / 4}\left(R_{d} / N\right)^{1 / 2}\left(10^{-20} \pi_{e}\right)^{1 / 2} \quad(\mathrm{cV})
$$

For the four-coil per corner case, considering plasmas with mid-10 $18 \mathrm{~m}^{-3}$ density and $R_{0}=0.4 \mathrm{~m}$, the threshold energy is about $30 \mathrm{eV}(10 \mathrm{eV}$ ) at the plasma center (edge). For the eight-coil per corner case, $T_{\mathrm{L}}=125 \mathrm{eV}$ at the plasma edge and is several orders of magnicude larger at the plasma center.

The eritical energy is defined as the energy above which particles will reach the wall if they are trapped in the ripple. When a particle is trapped in a ripple $\left(T>T_{\mathrm{t}}\right)$, its guiding center drifts along a contour of constant $B$. Because mod- $B$ contours are not closed in a toroidal field, the particles are not confined unless they are scatiered out of the ripple loss region before they can reach the wall. Thus, the critical cnergy can be determined by setting $\tau_{e n}$ (scattering) $=$ $\tau(\mathrm{drift})=a / v_{\text {drift, }}$, where $v_{\text {drin }}=T / e B R_{\sigma}$. For ions, this gives a critical energy (in mks units with $T$ in electron-volts) of

$$
T_{\cdot i}=1.6 \times 10^{3}\left(B R_{o} a / \delta\right)^{2 / 5}\left(10^{-20} n_{e}\right)^{2 / 3} \quad(\mathrm{eV})
$$

The critical energy is a factor of $\left(m_{i} / m_{e}\right)^{1 / 5}(\approx 4.5$ for a hydrogen plasma) larger for electrons. Again, considering the four-coil per corner case, $T_{-,}(r-0)=600 \mathrm{eV}$ and $T_{.}(r=a)=250 \mathrm{eV}$. Corresponding electron temperatures are higher (by a factor of -4 .5). For ail eight-coil per corner case, $T_{\bullet, i}(r-a)=1.5 \mathrm{keV}$ and $T_{\cdot, j}(r=0) \gg$ $T_{. j}(r=a)$.

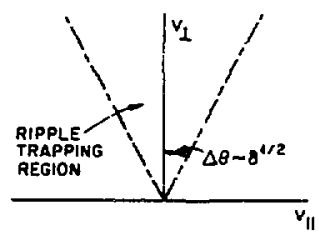

(a)

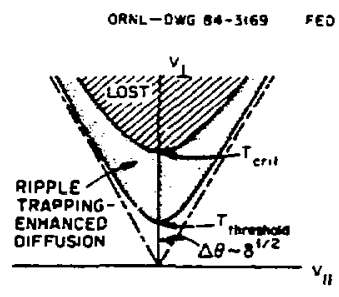

(b)
Fig. 9. (a) Ripple loss region and (b) critical energies. 
The critical energy previously defined is for a zero electric field. In the presence of a finite electric field, if the particles are trapped in the ripple and if their energy exceeds $T_{.}\left(T>T_{0}\right)$, the particle orbits are cot necessarily open because of the $\vec{E} \times \overrightarrow{\vec{s}}$ precessional drift, which balances the vertical (toroidal) drift. ${ }^{2}$ The shift in particle drift orbits is $\Delta x=v_{\text {drin }} / \Omega$, where $\Omega-\left(T / e B R_{c}\right)+(E / B)$ is the poloidal drift velocity with $R_{c}=-(\partial \ln B / \partial r)^{-1}$. For particle orbits to be closed, $\Delta x \leqslant a / 2$, or, conversely, for particies trapped in the ripple to drift to the wall, $\Delta x \geq a / 2$. Combining all gives a critical energy in the presence of an electric field above which particles will reach the wall:

$$
T_{\cdot c} \geqslant\left(\frac{e E a}{r}\right]\left[\left[\frac{1}{R_{0}}\right)-\left(\frac{a}{r R_{e}}\right)\right]^{-1} .
$$

For weak ripples, $\boldsymbol{r} \boldsymbol{R}_{\boldsymbol{c}}$ in the corner is very large (approaches infinity). With approximately $E=\Delta \phi / 2_{E}$, where $\ell_{E}$ is the electric field scale length (which is on the order of plasma radius), we have

$$
T_{\cdot E} \geqslant\left(a / \ell_{E}\right)\left(R_{N} / a\right) \Delta \phi=\Delta \phi / \epsilon
$$

Here $\epsilon$ is the inverse aspect ratio $(\epsilon-1 / 3-1 / 5)$. Thus, only if $T_{\cdot E} \geq(3-5) \Delta \phi$ will particles be directly lost. Combining all, we define

$$
\begin{aligned}
& T_{\text {criti }}=\max \left(T_{\cdot j} ; T_{\cdot E}\right), \\
& T_{\text {crile }}=\max \left(T_{\cdot, E} ; T_{\cdot E}\right) .
\end{aligned}
$$

\section{Ripple-Enhanced Diffesion-Simple Eutrantes}

From the critical energies $\left(T_{\mathrm{th}}, T_{\mathrm{cmi}}\right)$ defined earlier, we can see that the ripple collisionality regime can be divioed into three regions (Fig. 10). The upper collision frequency point $\left(y>v N \delta^{3 / 2} / R_{0}\right)$, above which transport losses diminish to zero, is the point at which the plasma particle temperature decreases below the threshold energy $\left(T<T_{t b}\right)$. In the middle range of the collisionality regime $(p \vee \delta / R a<,<$ $v N \delta^{3 / 2} / R_{o}$, where $\rho$ is the gyroradius), transport coeflicients scale as $\nu^{-I}$ because the particles are scattered out of the loss region before they can drift out of the device. To convey the spirit of simple diffusion estimates, we give a rough picture of the random walk process.

The average step-size taken by a ripple-t-Lpped particle is $(\Delta x)^{\delta} \sim v_{\text {drit }} \cdot \tau_{\text {ff }}^{\delta}=v_{\text {drif }} \cdot\left(\delta / v_{q_{0}}\right)$. The frequency with which such steps are taken is ${ }^{\prime}$ eff $=v_{0} \delta$. The fraction of particles participating in this ripple trapping is $=\delta^{1 / 2}$. Thus, the diffusion coefficient associated with ripple trapping is

$$
D^{\delta}-\left[(\Delta x)^{\delta}\right]^{2} \cdot v_{e f f} \cdot f^{\prime}-\delta^{3 / 2} v_{d \text { drin }}^{2} r_{90} .
$$

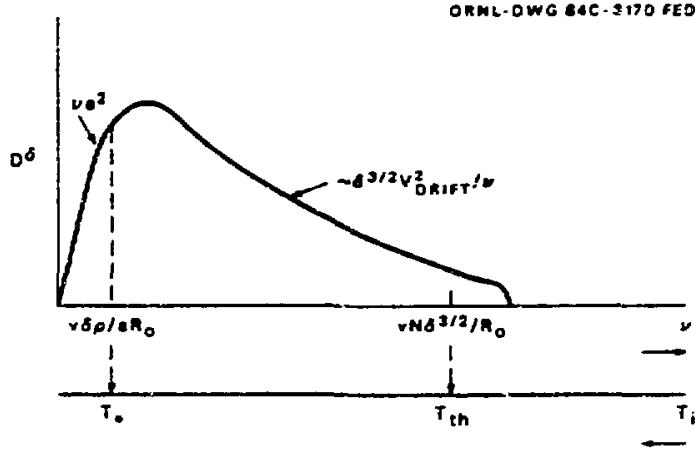

Fig. 10. Ripple collisionality regime.

Finally, in the absence of electric fields, at very low collision frequencies ( $\left.v<\rho v \delta / R_{-\infty}\right)$, the particle temperature increases above the critical energy, and all particles trapped in the ripples will drift to the wall without being seattered out of the loss region. The corresponding diffusion coefficient is $D-a_{50} a^{2}$.

\section{Comparison with Neoctescical Losses}

Here we compare the ripple-enhanced diffusion coeficients with the conventional EBT neoclassical diffusion coefficients to determine the allowable range for the magnitude of the field modulations (ripples). The neoclassical diffusion cocfficient for EBT is given by

$$
D^{N C}-(\Delta x)^{2}\left[v /\left(1+\nu^{2} / n^{2}\right)\right]
$$

where $\Delta x=v_{\text {drit }} / \Omega$ and $\nu=v_{90}$. In the collisionless regime $(v / \Omega<+1)$,

$$
D^{N C}-v_{90}(\Delta x)^{2} \sim \nu_{90}\left(v_{d r i R} / R\right)^{2} .
$$

In order for $D^{d}<D^{N C}$, that is,

$$
\left.\delta^{3 / 2} v_{\mathrm{driN}}^{2} / v_{90}<v_{90}\left(v_{\mathrm{drin}} / \mathrm{M}\right)\right)^{2} \text {, }
$$

we find $\varepsilon^{3 / 2}<(v / \Omega)^{2}$ or $\delta<(v / \Omega)^{4 / 3}$. For $y / \Omega \sim 0.1$, representative of present-day experiments, the magnitude of the ripple well depth should be $\delta<4.5 \%$ in order for ripple losses not to be doninant. For very low collision frequencies $(v / \Omega \sim 0.01$, representative of reactor-like piasmas), one requires $\delta<0.5 \%$. We see from this example that for the four-coil per corner case the ripple lowes will dominate. However, for the eight-coil per comer case the overall enhanced losses due to ripple will have a negligible effect on plasma confinement. 
We note that the estimates given here for ripple magnitudes are somewhat pessimistic due to aeglect of (1) the effect of poloidal variations in ripple magnitude and (2) the influence of the ambipolar electric field, both of which play an important role.

\section{ACKNOWLEDGMENT}

The authors would like to thank D. K. Lee for help in numerical calculations for field errors and in generating Figs. 4 and 5.

\section{RFFERENCES}

1. L. W. OWEN, D. K. LEE, and C. L. HEDRICK, "ElMO Bumpy Square," in Advanced Bumpy Torus
Concepts-Proceedings of the Workshop, CONF-830758, Oak Ridge National Laboratory (1983).

2. N. A. UCKAN et al., "ELMO Bumpy Square," ORNL/TM-91 10, Oak Ridge National Laboratory (1984).

3. T. UCKAN et al, "Field Errors in EBT and Their Effects on the Ambipolar Potential," ORNL/TM-7912, Oak Ridge National Laboratory (1981); T. UCKAN, "Fiekd Error Measurements on EBT," Bull. Am. Phys. Soc. 27, 1115 (1982).

4. T. UCKAN, -Inherent Magnetic Field Error Measurements in ELMO Bumpy Torus," Rev. Sci. Instrum. 56, (1985).

5. N. A. UCKAN et al. "Effects of the Poloidal Variation of the Magnetic Field Ripple on Enhanced Heat Transpors in Tokamaks," ORNL/TM-5438, Oak Ridge National Laboratosy (1976).

\section{DISCLAIMER}

This report was prepared as an account of work sponsored by an agency of the United States Government. Neither the United States Government nor any agency thereof, nor any of their employees, makes any warranty, express or implied, or assumes any legal liability or responsibility for the accuracy, completeness, or usefulness of any information, apparatus, product, or process disclosed, or represents that its use would not infringe privately owned rights. Reference herein to any specific commercial product, process, or service by trade name, trademark, manufacturer, or otherwise does not necessarily constitute or imply its endorsement, recommendation, or favoring by the United States Government or any agency thereof. The views and opinions of authors expressed herein do not necessarily state or reflect those of the United States Government or any agency thereof. 